\title{
Drug Burden Index in older adults: theoretical and practical issues
}

This article was published in the following Dove Press journal:

Clinical Interventions in Aging

9 September 2014

Number of times this article has been viewed

\section{Lisa Kouladjian' \\ Danijela Gnjidic ${ }^{1,2}$ \\ Timothy F Chen ${ }^{2}$ \\ Arduino A Mangoni ${ }^{3}$ \\ Sarah N Hilmer'}

'Sydney Medical School, University of Sydney, Kolling Institute of Medical

Research and Departments of Clinical Pharmacology and Aged Care, Royal

North Shore Hospital, ${ }^{2}$ Faculty of Pharmacy, University of Sydney, Sydney, NSW, ${ }^{3}$ Department of Clinical Pharmacology, School of Medicine, Flinders University and Flinders Medical Centre, Bedford Park, SA, Australia

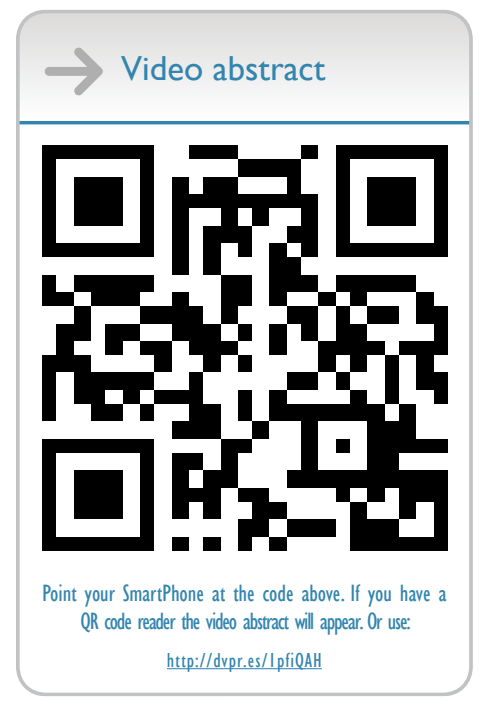

Correspondence: Lisa Kouladjian Clinical Pharmacology and Aged Care, Level 12 Kolling Building, Royal North Shore Hospital, Reserve Road, St Leonards, NSW, Australia 2065

Tel +6I 299264934

Fax +6I 299264053

Email lisa.kouladjian@sydney.edu.au
Abstract: Anticholinergic and sedative medications are commonly used in older adults and are associated with adverse clinical outcomes. The Drug Burden Index was developed to measure the cumulative exposure to these medications in older adults and its impact on physical and cognitive function. This narrative review discusses the research and clinical applications of the Drug Burden Index, and its advantages and limitations, compared with other pharmacologically developed measures of high-risk prescribing.

Keywords: Drug Burden Index, anticholinergics, sedative medications, high-risk prescribing, older adults, pharmacological risk assessment tools, deprescribing

\section{Clinical scenario}

An 81-year old Caucasian female presents with complaints of a dry mouth and morning sedation. The patient lives alone in a dwelling adjacent to her daughter and son-in-law, and has also noted a decreased ability to complete her daily activities due to fatigue. Her past medical history includes hypertension, urinary incontinence, insomnia, and osteoarthritic pain. Her medications include irbesartan $300 \mathrm{mg}$ daily, darifenacin $15 \mathrm{mg}$ daily, temazepam $7.5 \mathrm{mg}$ at night, and acetaminophen/codeine $300 \mathrm{mg} / 15 \mathrm{mg}$ two tablets three times daily. Her physician referred her for medication review by a pharmacist as part of the Medication Therapy Management pharmacy practice program. ${ }^{1}$

\section{Introduction}

The proportion of older people in the population is increasing globally. It is estimated that by $2050,22 \%$ of the total world's population will be aged over 60 years. ${ }^{2}$ Current evidence shows that older people are the largest per capita consumers of medications, especially the oldest old (aged over 84 years). ${ }^{3}$ The rise in polypharmacy (use of five or more medications) is driven by the growth of an aging and increasingly frail population with multiple chronic diseases. ${ }^{4}$

Medication management for older adults is fast becoming a challenge for health care professionals, and establishing the balance between the benefits and risks of medication use is often difficult. Many studies have highlighted the key role of regular assessment of medication regimens by primary health care professionals. According to the US Center for Medicare and Medicaid Services, it is estimated that polypharmacy costs US\$50 billion annually, ${ }^{5}$ and hence better evidence-based management approaches are essential to overcome polypharmacy and optimize medication use in older adults.

Some of the most commonly prescribed medications in older people include those

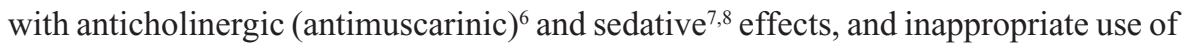
these medications is associated with adverse outcomes. ${ }^{9}$ For example, studies in older 
adults have concluded that high use of anticholinergic medications is associated with poor cognitive function ${ }^{10,11}$ and functional ${ }^{11}$ impairments. Many measures have been established to assess exposure to these types of medication in older adults, and each measure has its own role, advantages, and disadvantages.

This narrative review aims to evaluate and summarize the theoretical and practical aspects of the Drug Burden Index (DBI), ${ }^{12}$ a pharmacological risk assessment tool that measures the burden of anticholinergic and sedative medications in older adults. Specifically, it analyzes the effects of these medications in older adults, summarizes the development of the DBI, discusses the evidence supporting its utilization in practice, and compares the DBI with other pharmacologically developed models that measure anticholinergic or sedative exposure in older adults.

\section{Search strategy}

A search was conducted in Medline and PubMed using the following search terms: "Drug Burden Index", "anticholinergics", "sedatives", "scales", "index", "burden", "model”, "older people", "aged", and "aging". Published articles were reviewed starting from January 2000, and citations in reviews and research articles were also searched.

\section{Impact of anticholinergic and sedative medications on older adults}

Anticholinergic and sedative medications are used widely among older adults. Overuse of these medications has been associated with adverse drug events, some of which may limit physical and cognitive function. ${ }^{13}$ The muscarinic receptor blocking action of anticholinergics may cause dry mouth, constipation, blurred vision, increased heart rate, ${ }^{14}$ and confusion. ${ }^{9}$ Benzodiazepines, antipsychotics, antidepressants, opioid analgesics, ${ }^{15}$ and other drugs acting on the central nervous system can cause sedation, delirium, and impaired functional and cognitive status. Anticholinergic and sedative medications are prescribed in older adults to treat medical conditions that usually occur later in life, such as urinary incontinence, sleep and pain disorders, dementia, chronic obstructive pulmonary disease, and mental illness. However, evidence suggests that often the benefits do not justify the risks for some medications in older adults, for example chronic sedative medication use for insomnia. ${ }^{16}$ There is also significant evidence of an association between anticholinergic medications and neuropsychiatric adverse drug events in older adults. ${ }^{6}$ Evidence further suggests that clinicians may be less aware that some medications used in older adults, that are not prescribed for their anticholinergic properties, have anticholinergic effects, peripherally and centrally; ${ }^{9}$ for example, amitriptyline or doxepin for major depressive disorder. This is not surprising, as more than 600 drugs have been shown to have some degree of anticholinergic activity in vitro, with 14 of the 25 medications most commonly prescribed for older adults (in Italy) having detectable anticholinergic effects centrally. ${ }^{17}$ Furthermore, there is no gold standard definition for medications with anticholinergic effects for clinicians to refer to. ${ }^{18}$ As a consequence of multimorbidity and older age, many medications that act on the central nervous system and have sedative properties may result in depressive symptoms, impaired muscle strength, and worsen cognition and respiratory depression. This in turn may result in falls, fractures, hospitalization, and institutionalization. ${ }^{19}$ Additionally, several anticholinergic medications also have sedative properties, for example, olanzapine or quetiapine, making older people who are using medications from both classes, and who are already at risk from polypharmacy, more vulnerable to adverse drug events and their consequences.

\section{Development of the Drug Burden Index}

The DBI was developed and published in 2007 by Hilmer et $\mathrm{al}^{12}$ and measures the effect of cumulative exposure to both anticholinergic and sedative medications on physical and cognitive function in older adults. The DBI calculation was also intended to be an evidence-based risk assessment tool to guide prescribing in older people. It was proposed that the DBI calculation could be implemented in prescribing software to inform prescribers of the likely functional implications of an older patient's total exposure to these high-risk medications. A pharmacological equation was postulated by maintaining a classical dose-response relationship (Figure 1). The researchers hypothesized that the cumulative effect of anticholinergic and sedative medications would be linear, and a simple additive model was used to establish the total anticholinergic and sedative burden. The dose of each anticholinergic and sedative medication was used to determine a score from 0 to 1 for each drug in these classes. The relationship between an individual's DBI and their physical and cognitive performance was then evaluated in a cohort of community-dwelling older adults. It was shown that each additional unit of DBI had a negative effect on physical function similar to that of three additional physical morbidities. $^{12}$ 


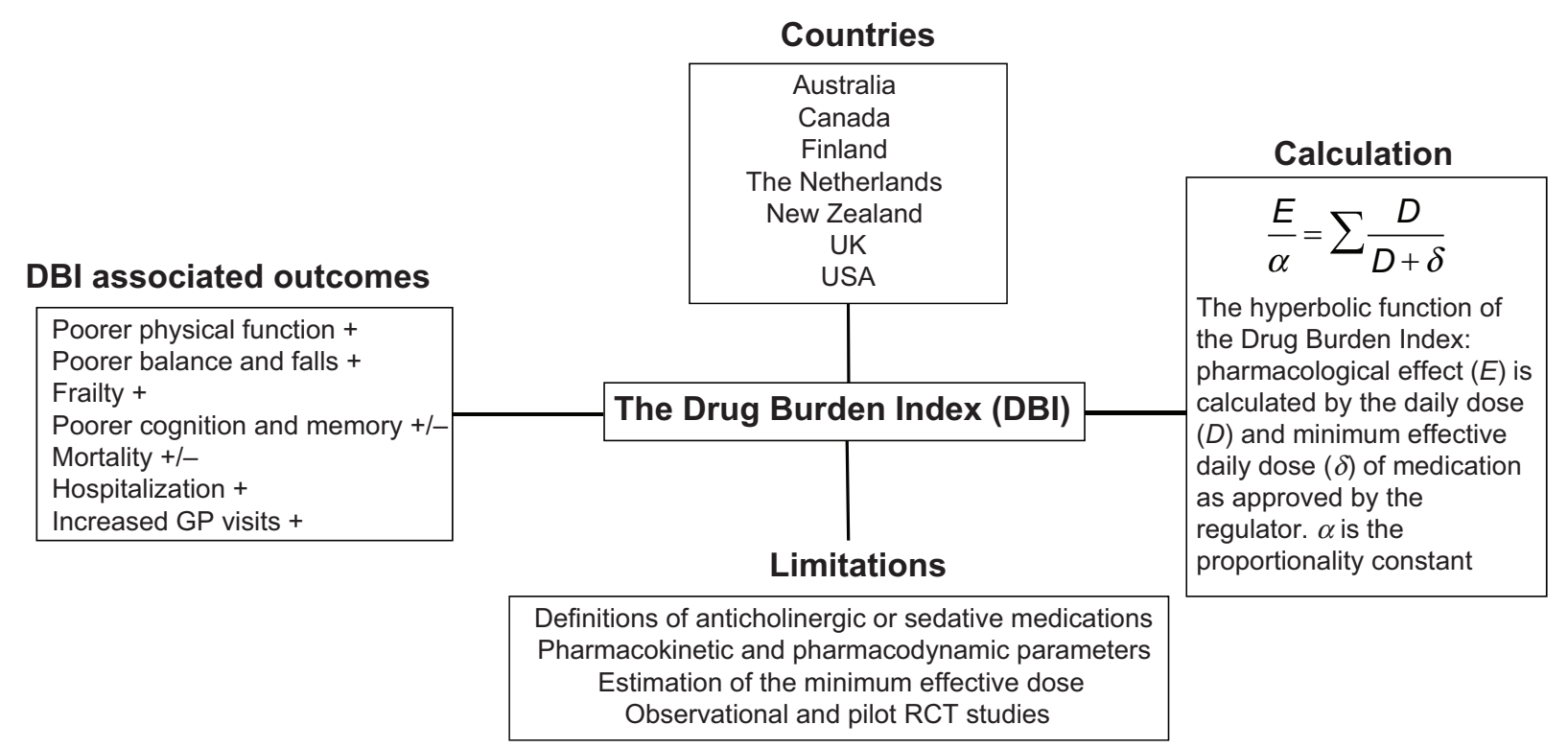

Figure I Summary of aspects of the DBI.

Notes: +, positive association; $+/-$, inconsistent association.

Abbreviations: RCT, randomized controlled trial; GP, general practitioner.

Mathematically, the drug burden is calculated for every patient according to the formula Total Drug Burden = $\mathrm{B}_{\mathrm{AC}}+\mathrm{B}_{\mathrm{S}}$, where $\mathrm{B}$ indicates burden, $\mathrm{AC}$ indicates anticholinergic, and $\mathrm{S}$ indicates sedative. Assuming that the anticholinergic and sedative effects of different drugs are additive in a linear fashion, ${ }^{20}$ it was postulated that $\mathrm{B}_{\mathrm{AC}}$ and $\mathrm{B}_{\mathrm{S}}$ may be proportional to a linear additive model of pharmacological effect $(E)$. This led to the formation of the equation,

$$
\frac{E}{\alpha}=\sum \frac{D}{D+D R_{50}}
$$

where $\alpha$ is a proportionality constant, $D$ is the daily dose, and $D R_{50}$ is the daily dose required to achieve $50 \%$ of maximal contributory effect at steady state. Furthermore, as the general $D R_{50}$ of anticholinergic and sedative effect is not identifiable and doses need to be normalized, the $D R_{50}$ was estimated as the minimum recommended daily dose $(\delta)$ as listed by the medication product information approved by the US Food and Drug Administration (Table 1). As there are some differences between countries in the $\delta$ of medications, subsequent studies using the DBI have used the minimum recommended daily dose in the study population setting. Besides being influenced by regulatory factors, the minimum daily dose accounts for additional factors that may influence drug effects such as genetics, ethnicity, diet, and environment. This flexibility allows the tool to be tailored for specific countries, for example, diazepam has a minimum registered/licensed dose of $4 \mathrm{mg}$ in the USA and $5 \mathrm{mg}$ in Australia.

The DBI is one of the few cumulative medication exposure measures that considers the dose. Furthermore, medications that are reported to be both sedative and anticholinergic are considered as anticholinergic to prevent double entries,

Table I Example of mathematical calculation of the Drug Burden Index (using clinical scenario)

\begin{tabular}{|c|c|c|c|}
\hline Medications in clinical scenario & Daily dose (D) & Minimum recommended daily dose $(\delta)$ & Individual DBI \\
\hline Irbesartan 300 mg, daily & $300 \mathrm{mg}$ & $75 \mathrm{mg}$ (no anticholinergic or sedative effects) & 0 \\
\hline Darifenacin 15 mg, daily & $15 \mathrm{mg}$ & $7.5 \mathrm{mg}$ (anticholinergic effects) & 0.67 \\
\hline Temazepam 7.5 mg, at night & $7.5 \mathrm{mg}$ & 7.5 mg (sedative effects) & 0.50 \\
\hline Acetaminophen $300 \mathrm{mg}, 2$ tablets tds & $\mathrm{I}, 800 \mathrm{mg}$ & $300 \mathrm{mg}$ (no anticholinergic or sedative effects) & 0 \\
\hline Codeine $15 \mathrm{mg}, 2$ tablets tds & $90 \mathrm{mg}$ & I 20 mg (sedative effects) & 0.43 \\
\hline
\end{tabular}

Total DBI calculation

$D B I=\sum \frac{D}{D+\delta}$

Notes: This is an example of a medication regimen for an older adult in the USA; minimum daily dose as recommended by the US Food and Drug Administration. Abbreviations: tds, three times a day; DBI, Drug Burden Index. 
and therefore a separate anticholinergic DBI can be calculated from the DBI. Originally, medications with clinically significant anticholinergic or sedative effects were identified by means of Mosby's Drug Consult ${ }^{21}$ and the Physicians' Desk Reference. ${ }^{22}$ Further observational studies have used various country-specific resources to identify these high-risk medications with similar findings, reflecting the adaptability of the equation to the investigation setting.

\section{Validation studies of the Drug Burden Index}

Increasing DBI exposure has been associated with poorer physical function, ${ }^{23-26}$ frailty, ${ }^{27}$ and falls. ${ }^{28,29}$ With regard to hospitalization, a higher DBI has also been associated with increased hospital days, increased hospitalization for delirium, and readmission to hospital (Table 2).

There have been mixed reports on the association of DBI with mortality and cognition. One study conducted in people living in residential care facilities in Australia reported a non-significant association between high DBI and mortality. ${ }^{30}$ A study conducted in the Netherlands on older patients admitted to hospital with hip fractures showed a significant univariate but not multivariate association of increasing anticholinergic component of the DBI with oneyear mortality. ${ }^{18}$ A population-based study of older people in New Zealand found that DBI exposure increased the risk of mortality, ${ }^{28}$ and a study in Finland found an association of high DBI exposure with mortality in people with and without Alzheimer's disease. ${ }^{31}$

Regarding cognition, the original validation study in the USA ${ }^{12}$ found that increasing DBI was associated with impaired cognition in community-dwelling older people when measured using the digit symbol substitution test; however, no associations were observed between DBI and cognition in a cohort of community-dwelling older men in Australia. ${ }^{32}$ Observational studies conducted in older adults from different international settings generate relatively consistent findings, confirming a trend towards prescribing of these high-risk medications and poorer physical function outcomes in older adults.

\section{Utility of the Drug Burden Index in practice}

Currently, most studies of DBI in clinical practice have been observational. Limited studies have investigated associations between pharmaceutical service provision, prescribing outcomes, and functional outcomes in older adults (Table 3). In Australia, Home Medicine Review and Residential
Medication Management Review are community-based collaborative services provided by general practitioners and pharmacists and fall under the Commonwealth-funded Medication Management Initiatives. ${ }^{33}$ Two retrospective analyses have investigated DBI associations before and after Home Medicine Review and Residential Medication Management Review services, respectively. Castelino et $\mathrm{al}^{34}$ concluded that the recommendations provided by a pharmacist as part of the service resulted in a significant reduction in the sum total of DBI scores for all patients. Similarly, Nishtala et al ${ }^{35}$ found that DBI scores of participants were significantly lower after the pharmacist-led Residential Medication Management Review intervention. In both studies, the DBI was used as a screening or evaluation tool to measure service provision, the quality of pharmacists' recommendations, and prescribing changes by general practitioners following uptake of recommendations. As these studies were retrospective in nature, it was not possible to assess changes in function and cognition of subjects. Additionally, these studies suggest that the DBI may be used to evaluate medication therapy management services, alongside other markers such as polypharmacy (five or more medications), hyperpolypharmacy (ten or more medications), ${ }^{27}$ or use of psychotropic medications (Omnibus Budget Reconciliation Act 1987) ${ }^{36}$ to demonstrate prescribing appropriateness and predict functional outcomes in older adults.

To date, only one interventional study has been conducted in the primary care setting using the DBI. This was a pilot cluster, randomized controlled trial in people aged $\geq 70$ years living in retirement villages. ${ }^{37}$ For participants in the intervention group general practitioners were provided with patient-specific information on their patient's DBI and its health/functional implications, and were asked to consider cessation or dose reduction of anticholinergic and sedative medications contributing to DBI. For those in the control group, general practitioners were provided with their patient's medication list only. Amongst those with DBI $>0$ at baseline, DBI was reduced in $32 \%$ of the intervention group and in $19 \%$ of control participants. ${ }^{37}$ However, this approach only targeted general practitioners, and may reflect the poor effectiveness of the intervention design. Recent reviews state the lack of evidence for clinical effectiveness and sustainability of varying interventions to reduce polypharmacy. ${ }^{38,39}$ Further studies have demonstrated that multidisciplinary interventions using various intervention combinations (such as educational material, computerized support systems, medication reviews by pharmacists) are more effective than single interventions, such as that used 


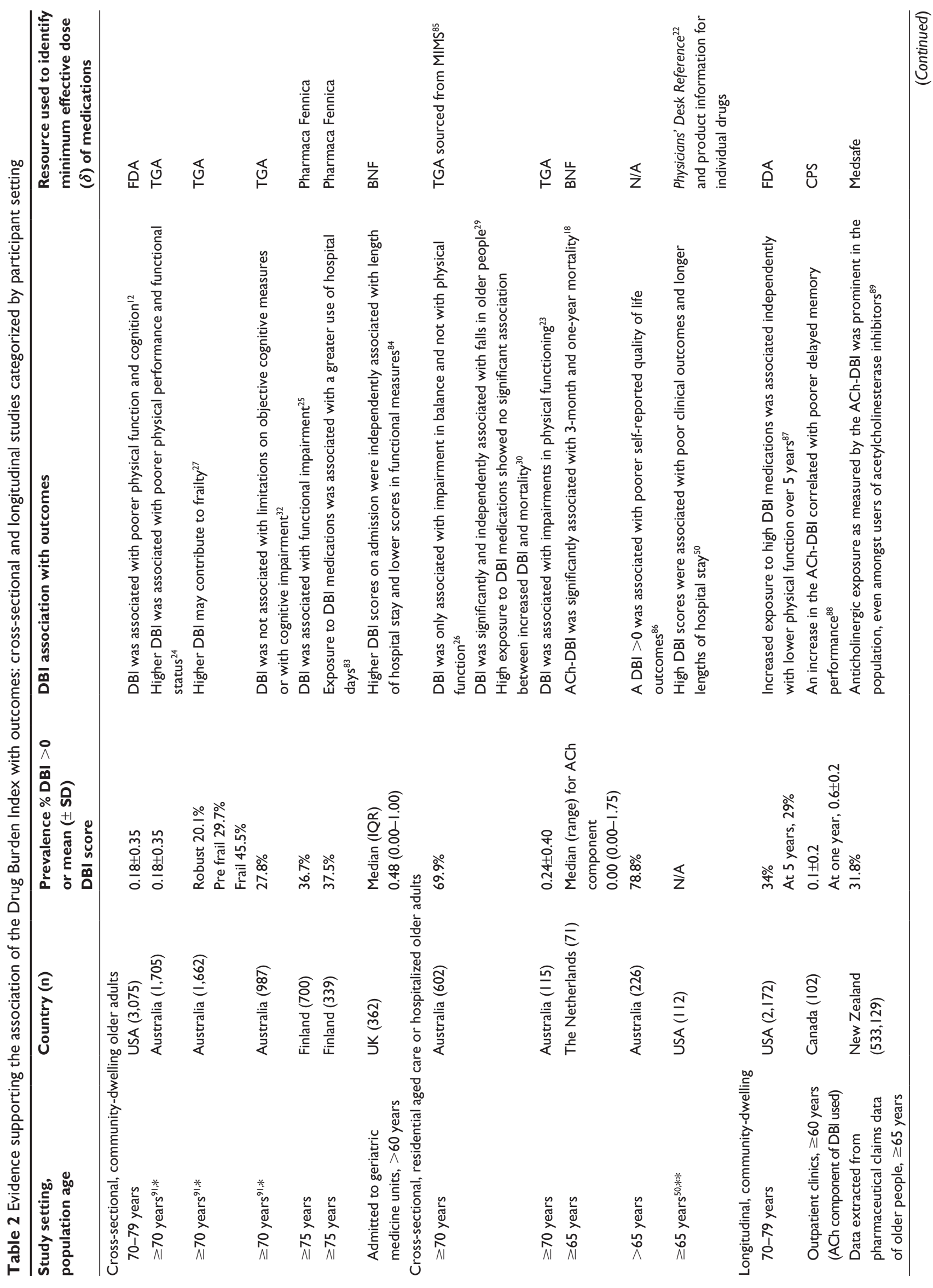




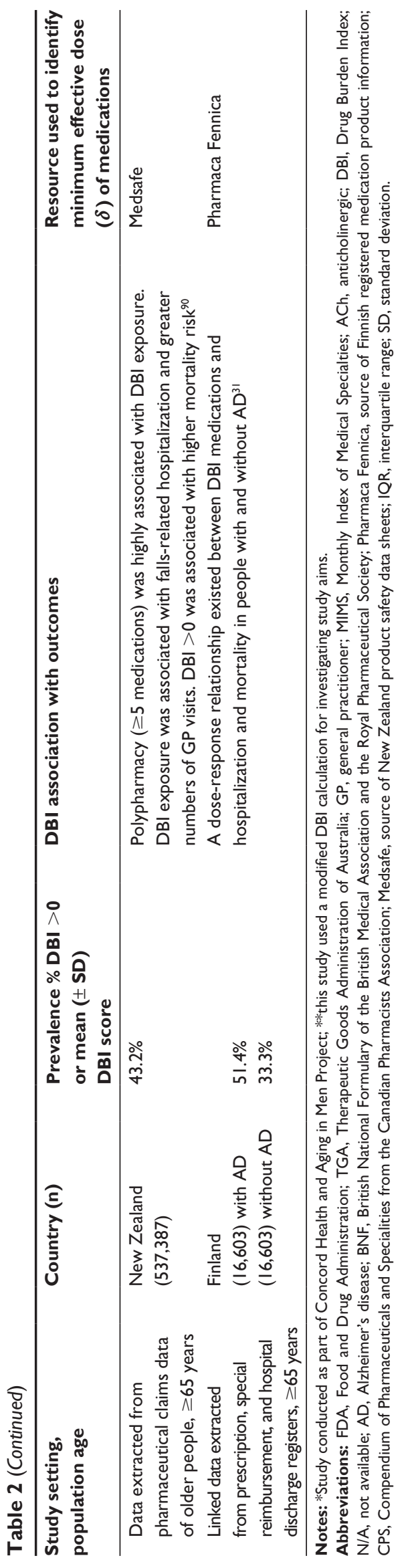

in the pilot randomized controlled trial,,${ }^{37}$ at reducing inappropriate prescribing in older people. ${ }^{39,40}$

Conclusively, these studies demonstrate the need for further research into multidisciplinary and multifactorial interventions using the DBI to evaluate prescribing and functional outcomes in older adults.

\section{Theoretical aspects of the Drug Burden Index}

Given its novelty and adaptability, the DBI has many theoretical applications in various settings for evaluating appropriate prescribing of anticholinergic and sedative medications in older adults. The original study by Hilmer et al ${ }^{12}$ suggested that the DBI "by means of prescribing software" can inform clinicians of the implications of these high-risk medications in older people. Computerized decision support systems and computerized advice on drug dosage have been shown to improve prescribing practices, but have a mixed impact on patient outcomes. ${ }^{41-43}$ A recent meta-analysis concluded that electronic decision support systems that provided recommendations to both patients and clinicians may improve the success of interventions. ${ }^{44}$ Currently, the DBI is being developed as a software for use by pharmacists and general practitioners during the medication review process. ${ }^{45}$

Furthermore, as the DBI has been validated across a number of countries, there may be many international applications of the DBI. Faure et al ${ }^{46}$ suggested that standardizing the DBI calculation with the defined daily dose, as determined by the World Health Organization, would result in a universal quality indicator in drug management, ie, the "international DBI". While there are concerns about the validity of the defined daily dose as an estimate of $D R_{50}{ }^{47}$ an international DBI would allow comparison of anticholinergic and sedative medication exposure and clinical outcomes in older patients globally, and could be applied to developing countries without national formularies.

\section{Considerations when interpreting the Drug Burden Index}

Like other pharmacological-based scales, the DBI has various limitations which must be considered when applying the tool in clinical settings. As discussed in the literature, it is difficult to determine exactly what constitutes an anticholinergic or sedative medication. There is no consensus on how to define an anticholinergic drug. ${ }^{20}$ Several scales have been developed by consensus panels and expert opinion. This may be difficult to justify unless the method used to arrive at consensus (for example, the Delphi method) is clearly stated for the purpose 


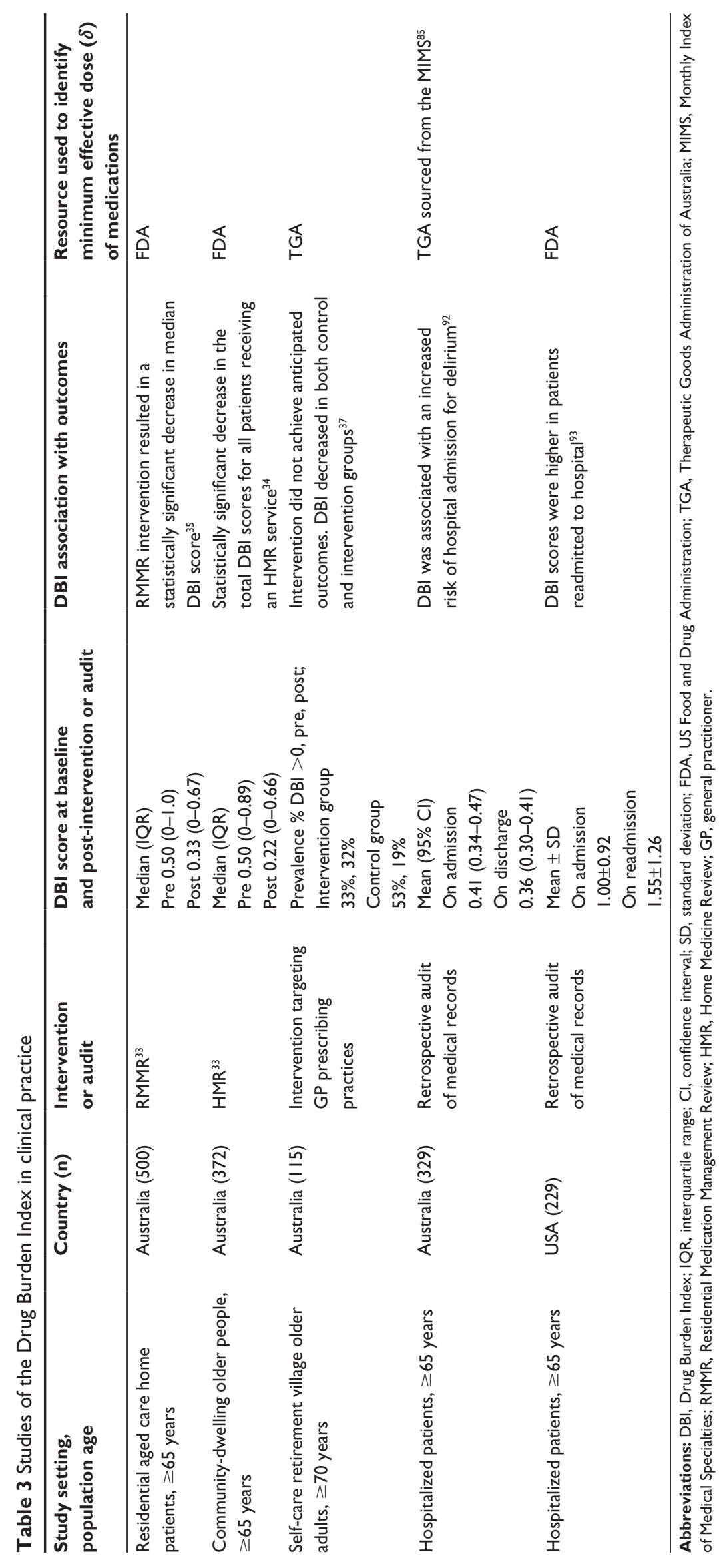


investigated, and conflicts of interest are put forward, so as not to introduce bias to the outcome. ${ }^{48}$ The DBI uses the drug monograph to determine whether a drug has an anticholinergic or sedative effect by considering aspects of the pharmacology and side effect profiles. However, this method is also subject to interpretation bias. For example, anticholinesterases do not form part of the anticholinergic component of the DBI, but are included in the sedative component, and hence may cause confusion when calculating the DBI for a patient.

In terms of the calculation itself, the $D R_{50}$ is estimated as the minimum efficacious dose according to the approved product information. However, it does not take into account the specific indication nor interpatient pharmacokinetic or pharmacodynamic variations. This estimate is based on the regulatory principle that a registered dose must have some efficacy and that the minimum dose is likely to have less than maximal efficacy. The minimum recommended daily dose $(\delta)$ was used as an estimate of the dose needed to achieve $50 \%$ of the maximum effect. The accuracy of the estimate of minimum efficacious dose may differ among drugs and subjects with different pharmacokinetic and pharmacodynamic characteristics. The DBI also assumes a linear dose-response relationship between drug classes. All medications with clinically relevant sedative or anticholinergic properties are considered equivalent, which may overestimate or underestimate the effect of the medication on the patient clinically.

Furthermore, previous studies considering the DBI have excluded the use of "as required" medications. Estimates of exposure to different dosing forms of medications (topical creams, eye drops, patches) have also not been clearly explained. This again may overestimate or underestimate the use of the anticholinergic or sedative medication of interest.

Drug response varies with basic physiological and pharmacological factors such as genetic variations, gender variations, and ethnicity. Additionally, in older adult populations, factors such as multimorbidity, polypharmacy, changes in organ function (such as liver or renal clearance), and frailty need to be considered when calculating medication burden. ${ }^{49}$ None of these factors are accounted for by the DBI calculation. Validation studies conducted in various countries and settings have demonstrated a wide range of prevalence of exposure to DBI medications, from $20 \%$ to $79 \%$ (Table 2). This variability may be related to varying definitions of anticholinergic and sedative medications sourced from monographs in different countries, specific sociodemographic or health status characteristics of participants, and factors related to the health care system influencing prescription of these medications. Furthermore, modifications to the DBI calculation may limit the validity and interpretation of the tool with important clinical outcomes. A recent study ${ }^{50}$ used a modified DBI calculation to conclude that higher DBI scores were associated with poor clinical outcomes and longer lengths of hospital stay. However, this study did not comment on associations with these clinical outcomes if the calculation was kept consistent with previous studies. The modified DBI may lead to overestimation or underestimation of the association of anticholinergic and sedative medication burden with important clinical outcomes in older adults.

Finally, as previously described, the evidence for the external validity and clinical applicability of the DBI is from observational studies and one pilot interventional study. Furthermore, several studies by Gnjidic et al ${ }^{23,27,32}$ were conducted in older male adults, and extrapolating data to females may be limited due to the differences in prescribing medications and variations in disease prevalence, functional impairment, and frailty between sexes.

\section{Comparison of Drug Burden Index with other evidence-based pharmacological tools}

Several measures of inappropriate prescribing have been developed with research on its prevalence, its association with adverse outcomes, and interventions to reduce this type of medication exposure. Various explicit and implicit inappropriate medication use scales, such as the Beers criteria, ${ }^{51}$ STOPP/START (Screening Tool of Older Person's Prescriptions/Screening Tool to Alert doctors to Right Treatment) criteria, ${ }^{52,53}$ Medication Appropriateness Index, ${ }^{54,55}$ and Australian Indicators, ${ }^{56,57}$ are primarily used as general prescribing indicator tools across all medication classes rather than specifically with anticholinergic and sedative medications. Several other scales have been developed for evaluating exposure to anticholinergic or sedative medications alone. Examples of the anticholinergic scales include the Anticholinergic Drug Scale, ${ }^{58}$ the Anticholinergic Burden Classification, ${ }^{59}$ Clinician-rated Anticholinergic Score, ${ }^{60}$ Anticholinergic Risk Scale, ${ }^{61}$ Anticholinergic Activity Scale, ${ }^{62}$ Anticholinergic Load Scale, ${ }^{63}$ and Anticholinergic Cognitive Burden Scale, ${ }^{64}$ whilst the scales to determine the cumulative effects of taking multiple medications with sedative properties include the Sedative Load Model, ${ }^{65,66}$ the Sloane Model, ${ }^{67}$ the Central Nervous System Drug Model, ${ }^{68-70}$ and the Antipsychotic Monitoring Tool. ${ }^{71}$ However, when it comes to which scale or pharmacological indicator tool to apply for an older adult 
to make a decision related to medication management in clinical practice, selecting the right tool for the individual patient situation is challenging for health care professionals. Each anticholinergic and sedative pharmacological tool has specific advantages and disadvantages based on the original study objectives and populations. Furthermore, only a few scales have been used in randomized controlled trials to determine prescribing outcomes in older adults..$^{37,72}$

There is considerable variation among the anticholinergic scales ${ }^{14}$ which is not surprising since the methodologies used to calculate and develop these scales vary significantly (Table 4). Additionally, a recent study concluded that there was poor agreement between the Anticholinergic Drug Scale, Anticholinergic Risk Scale, and Anticholinergic Cognitive Burden Scale, and suggested the scales could not be applied to varying study settings unless consistently updated. ${ }^{73}$ Furthermore, it has been proposed that a definitive international list of anticholinergic medications is important to build credible screening tools and to translate research with these scales to associations with clinical outcomes in older adults. ${ }^{46}$ However, establishing this "definitive" list would be difficult, as there are conflicting data in the literature on what constitutes an anticholinergic drug. Compiling such a list would require standardization of the anticholinergic effects in a typical older adult who has varied pharmacological exposures and physiological functions, and is subjected to external factors such as genetics, ethnicity, diet and environment.

It has been argued that the gold standard for assessing a drug's anticholinergic effect remains the serum anticholinergic activity. ${ }^{58}$ However, a recent study found that clinical anticholinergic effects in patients were only partially associated with serum anticholinergic activity, ${ }^{18}$ adding to the complexity of defining an anticholinergic drug. Serum anticholinergic activity assays using peripheral blood may misrepresent anticholinergic effects centrally.

Similarly, in terms of sedative medications, universal definitions of what constitutes a sedative medication or a sedative load are lacking (Table 5). A review comparing four methods to quantify the effect of sedative medications on older people concluded that the usefulness of each method in clinical practice is yet to be determined. ${ }^{15}$ Pharmacological tools that are developed using various methods (Tables 4 and 5) should be applied to population settings originally described which investigated the outcomes they were designed to predict. Tools may be successively adapted according to medications available in the country investigated and revalidated in the new setting. This will more accurately reflect the impact of these medications on older adults in the setting investigated, but propose a challenge

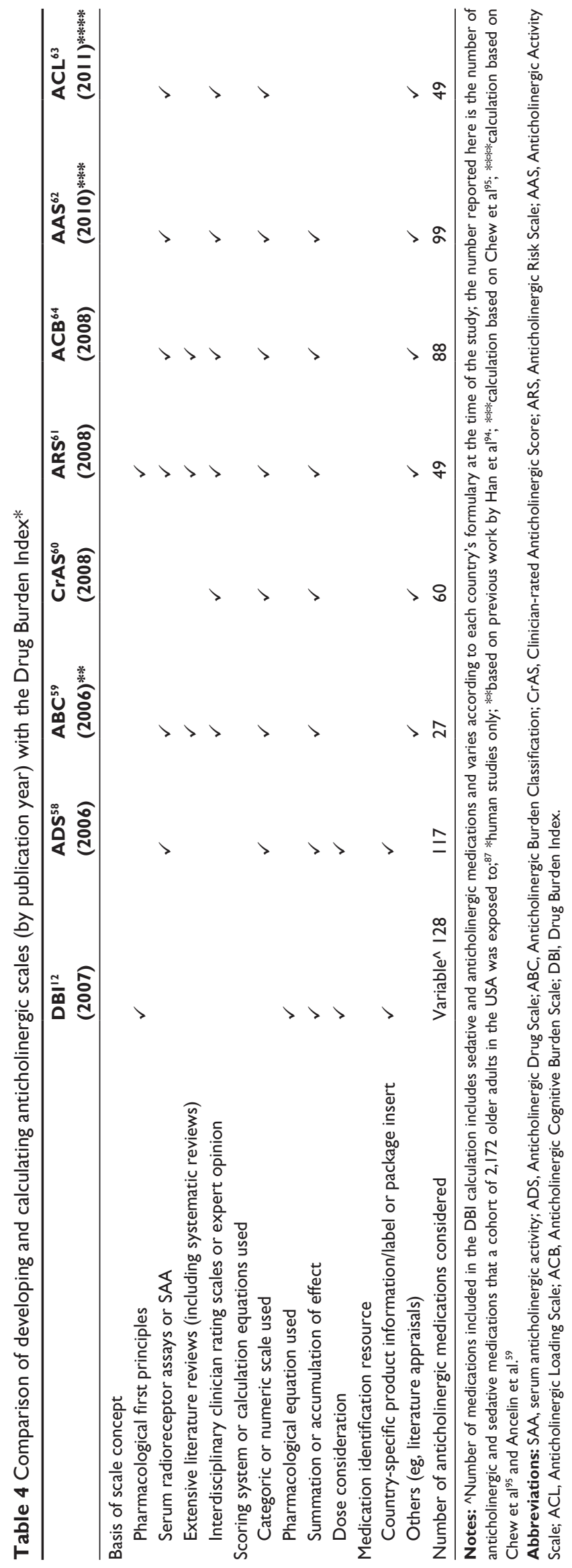


Table 5 Comparison of developing sedative scales (by publication year) with the Drug Burden Index*

\begin{tabular}{|c|c|c|c|c|}
\hline & DBI $(2007)^{12}$ & $\begin{array}{l}\text { Sedative } \\
\text { load }(2003)^{65,66}\end{array}$ & $\begin{array}{l}\text { Sloane et al } \\
(2008)^{67, * * *}\end{array}$ & $\begin{array}{l}\text { CNS drug } \\
(2009)^{68-70}\end{array}$ \\
\hline \multicolumn{5}{|l|}{ Basis of scale concept } \\
\hline Pharmacological first principles & $\checkmark$ & & $\checkmark$ & $\checkmark$ \\
\hline Extensive literature reviews (including systematic reviews) & & $\checkmark$ & & \\
\hline Interdisciplinary clinician rating scales or expert opinion & & $\checkmark$ & $\checkmark$ & \\
\hline \multicolumn{5}{|l|}{ Scoring system or calculation equations used } \\
\hline Categoric or numeric scales used & & $\checkmark$ & $\checkmark$ & $\checkmark$ \\
\hline Pharmacological equation used & $\checkmark$ & $\checkmark$ & $\checkmark$ & $\checkmark$ \\
\hline Summation or accumulation of effect & $\checkmark$ & $\checkmark$ & $\checkmark$ & $\checkmark$ \\
\hline Dose consideration & $\checkmark$ & & $\checkmark$ & $\checkmark$ \\
\hline \multicolumn{5}{|l|}{ Medication identification resource } \\
\hline Anatomical Therapeutic Classification System & & $\checkmark$ & $\checkmark$ & \\
\hline lowa Drug Information System Codes & & & & $\checkmark$ \\
\hline Country-specific product information/label or package insert & $\checkmark$ & $\checkmark$ & $\checkmark$ & \\
\hline Number of sedative medications considered & Variable^ $128^{87}$ & $340^{65}$ & 106 & $53^{70}$ \\
\hline
\end{tabular}

Notes: ^Number of medications included in the DBI calculation includes sedative and anticholinergic medications and varies according to each country's formulary at the time of the study; the number reported here is the number of anticholinergic and sedative medications that a cohort of 2,172 older adults in the USA was exposed to; *human studies only; **based on calculations from sedative load.

Abbreviations: DBI, Drug Burden Index; CNS, central nervous system.

to compare outcomes globally. Overall, an ideal pharmacological tool to investigate high-risk medication use in older adults in clinical practice should be accurate, reliable, easily accessible, easy-to-use, adaptable to the setting, based on current evidence, and provide evidence-based information that is consistently updated, to guide prescribing or drug withdrawal.

\section{Future: Drug Burden Index and deprescribing}

The term "deprescribing" has recently been discussed in the literature and describes the process of ceasing of unnecessary or harmful medications, especially in older adults. ${ }^{74,75}$ Deprescribing has been proposed as an important aspect of medication prescribing, monitoring, and use. ${ }^{76}$ The DBI, together with other scales, may have a clinical role in evaluating the medication load for a patient. Written reports of the evidence describing use of these high-risk medications and negative clinical outcomes can give prescribers the incentive to deprescribe inappropriate anticholinergic and sedative medications. Many studies have proposed use of single-active pharmacological scales in conjunction with medication review services, such as Medication Therapy Management or the Medication Management Initiative. ${ }^{77-79}$ Although this may facilitate action by health care professionals, such an approach focuses on anticholinergics or sedatives alone (and not in combination). A potential risk of this intervention is a shift from a patient-centered care approach when evaluating the benefits and risks of medications in older adults. While exposure to anticholinergic and sedative medications is associated with functional impairment in older adults, this does not dismiss other medication classes, such as anticoagulants, antiplatelets, and hypoglycemics, as high-risk. ${ }^{80}$

\section{Conclusion}

In conclusion, as the proportion of older people in the global population grows, and the prevalence of polypharmacy, multimorbidity, and adverse outcomes associated with these increases, there are various pharmacological tools to guide health care professionals to manage medications. The DBI is a novel pharmacological evidence-based tool which measures an individual's total exposure to anticholinergic and sedative medications and is strongly associated with functional impairment. Increasing DBI has been associated with poorer physical function, falls, frailty, hospitalization, and mortality (Figure 1). Future applications of the DBI include integration into practice through online software and prescribing tools, and incorporation into deprescribing studies. The DBI is one of many tools to guide prescribing in older adults, and has a specific role in measuring the functional burden of their medications. A patient-centered multidisciplinary approach to medication management, using multiple risk assessment tools combined with regular review of medication regimens, may provide the best approach to ensure the quality use of medicines for older people worldwide.

\section{Scenario resolution}

During Medication Therapy Management, the pharmacist conducted a comprehensive medication review. In the report 
back to the primary care physician, the pharmacist reported a DBI of 1.6 (Table 1), which translates to a negative effect on physical function on this patient greater than that of three additional physical comorbidities. ${ }^{12}$ Furthermore, this patient was at a high risk of falls. The medication-related action plan included deprescribing temazepam, discussing non-pharmacological strategies for insomnia, reassessing the need for high-dose darifenacin and reducing the dose, and replacing the acetaminophen/codeine combination with acetaminophen alone, while monitoring the osteoarthritic pain. These were also outlined in the patient's personal medication record. After one month, the dose of darifenacin was reduced and the codeine ceased. The patient's dry mouth and fatigue had resolved and she reported improvement in her activities of daily living. While initially reluctant to withdraw temazepam, the patient was now considering this, following direct-to-patient education about the risks of benzodiazepine use ${ }^{81}$ and in view of the improvements she had already experienced after reducing her anticholinergic and sedative medication load. ${ }^{82}$

\section{Disclosure}

The authors report no conflicts of interest in this work.

\section{References}

1. American Pharmacists Association; National Association of Chain Drug Stores Foundation. Medication therapy management in pharmacy practice: core elements of an MTM service model (version 2.0). J Am Pharm Assoc. 2008;48(3):341-353.

2. World Health Organisation. Ageing and life course: interesting facts about ageing. Geneva, Switzerland: World Health Organisation; 2012. Available from: http://www.who.int/ageing/about/facts/en/index.html\#. Accessed July 9, 2014.

3. Linjakumpu T, Hartikainen S, Klaukka T, Veijola J, Kivelä S-L, Isoaho R. Use of medications and polypharmacy are increasing among the elderly. J Clin Epidemiol. 2002;55(8):809-817.

4. Wise J. Polypharmacy: a necessary evil. BMJ. 2013;347:f7033.

5. Bushardt RL, Massey EB, Simpson TW, Ariail JC, Simpson KN. Polypharmacy: misleading, but manageable. Clin Interv Aging. 2008;3(2): 383-389.

6. Nishtala PS, Fois RA, McLachlan AJ, Bell JS, Kelly PJ, Chen TF. Anticholinergic activity of commonly prescribed medications and neuropsychiatric adverse events in older people. J Clin Pharmacol. 2009;49(10):1176-1184.

7. Rothberg MB, Herzig SJ, Pekow PS, Avrunin J, Lagu T, Lindenauer PK. Association between sedating medications and delirium in older inpatients. J Am Geriatr Soc. 2013;61(6):923-930.

8. Ineke Neutel C, Skurtveit S, Berg C. Polypharmacy of potentially addictive medication in the older persons - quantifying usage. Pharmacoepidemiol Drug Saf. 2012;21(2):199-206.

9. Bell JS, Mezrani C, Blacker N, et al. Anticholinergic and sedative medicines - prescribing considerations for people with dementia. Aust Fam Physician. 2012;41(1-2):45-49.

10. Fox C, Richardson K, Maidment ID, et al. Anticholinergic medication use and cognitive impairment in the older population: the Medical Research Council Cognitive Function and Ageing study. J Am Geriatr Soc. 2011;59(8):1477-1483.
11. Koyama A, Steinman M, Ensrud K, Hillier TA, Yaffe K. Long-term cognitive and functional effects of potentially inappropriate medications in older women. J Gerontol A Biol Sci Med Sci. 2014;69(4):423-429.

12. Hilmer SN, Mager DE, Simonsick EM, et al. A Drug Burden Index to define the functional burden of medications in older people. Arch Intern Med. 2007;167(8):781-787.

13. Feinberg M. The problems of anticholinergic adverse effects in older patients. Drugs Aging. 1993;3(4):335-348.

14. Duran CE, Azermai M, Vander Stichele RH. Systematic review of anticholinergic risk scales in older adults. Eur J Clin Pharmacol. 2013; 69(7):1485-1496.

15. Taipale HT, Hartikainen S, Bell JS. A comparison of four methods to quantify the cumulative effect of taking multiple drugs with sedative properties. Am J Geriatr Pharmacother. 2010;8(5):460-471.

16. Glass J, Lanctot KL, Herrmann N, Sproule BA, Busto UE. Sedative hypnotics in older people with insomnia: meta-analysis of risks and benefits. BMJ. 2005;331(7526):1169.

17. Cancelli I, Beltrame M, Gigli GL, Valente M. Drugs with anticholinergic properties: cognitive and neuropsychiatric side-effects in elderly patients. Neurol Sci. 2009;30(2):87-92.

18. Mangoni AA, van Munster BC, Woodman RJ, de Rooij SE. Measures of anticholinergic drug exposure, serum anticholinergic activity, and all-cause postdischarge mortality in older hospitalized patients with hip fractures. Am J Geriatr Psychiatry. 2013;21(8):785-793.

19. Craig D, Passmore AP, Fullerton KJ, et al. Factors influencing prescription of CNS medications in different elderly populations. Pharmacoepidemiol Drug Saf. 2003;12(5):383-387.

20. Kersten H, Wyller TB. Anticholinergic drug burden in older people's brain - how well is it measured? Basic Clin Pharmacol Toxicol. 2014; 114(2):151-159.

21. Nissen D. Mosby's Drug Consult. St Louis, MO, USA: Mosby Inc; 2004.

22. Duplay D. Physicians' Desk Reference. 58th ed. Montvale, NJ, USA: Thomson PDR; 2004.

23. Gnjidic D, Le Couteur DG, Abernethy DR, Hilmer SN. Drug Burden Index and Beers criteria: impact on functional outcomes in older people living in self-care retirement villages. J Clin Pharmacol. 2012;52(2): $258-265$.

24. Gnjidic D, Cumming RG, Le Couteur DG, et al. Drug Burden Index and physical function in older Australian men. Br J Clin Pharmacol. 2009;68(1):97-105.

25. Gnjidic D, Bell JS, Hilmer SN, Lonnroos E, Sulkava R, Hartikainen S. Drug Burden Index associated with function in community-dwelling older people in Finland: a cross-sectional study. Ann Med. 2012;44(5): 458-467.

26. Wilson NM, Hilmer SN, March LM, et al. Associations between Drug Burden Index and physical function in older people in residential aged care facilities. Age Ageing. 2010;39(4):503-507.

27. Gnjidic D, Hilmer SN, Blyth FM, et al. High-risk prescribing and incidence of frailty among older community-dwelling men. Clin Pharmacol Ther. 2012;91(3):521-528.

28. Nishtala PS, Narayan SW, Wang T, Hilmer SN. Associations of Drug Burden Index with falls, general practitioner visits, and mortality in older people. Pharmacoepidemiol Drug Saf. 2014;23(7):753-758.

29. Wilson NM, Hilmer SN, March LM, et al. Associations between Drug Burden Index and falls in older people in residential aged care. $J \mathrm{Am}$ Geriatr Soc. 2011;59(5):875-880.

30. Wilson NM, Hilmer SN, March LM, et al. Associations between Drug Burden Index and mortality in older people in residential aged care facilities. Drugs Aging. 2012;29(2):157-165.

31. Gnjidic D, Hilmer SN, Hartikainen S, et al. Impact of high risk drug use on hospitalization and mortality in older people with and without Alzheimer's disease: a national population cohort study. PLoS One. 2014;9(1):e83224.

32. Gnjidic D, Le Couteur DG, Naganathan V, et al. Effects of Drug Burden Index on cognitive function in older men. $J$ Clin Psychopharmacol. 2012;32(2):273-277. 
33. Community Pharmacy Agreement. Medication Management Initiatives. Available from: http://5cpa.com.au/programs/medication-managementinitiatives/home-medicines-review/. Accessed July 10, 2013.

34. Castelino RL, Hilmer SN, Bajorek BV, Nishtala P, Chen TF. Drug Burden Index and potentially inappropriate medications in communitydwelling older people: the impact of Home Medicines Review. Drugs Aging. 2010;27(2):135-148.

35. Nishtala PS, Hilmer SN, McLachlan AJ, Hannan PJ, Chen TF. Impact of residential medication management reviews on Drug Burden Index in aged-care homes: a retrospective analysis. Drugs Aging. 2009;26(8): 677-686.

36. Lantz MS, Giambanco V, Buchalter EN. A ten-year review of the effect of OBRA-87 on psychotropic prescribing practices in an academic nursing home. Psychiatr Serv. 1996;47(9):951-955.

37. Gnjidic D, Le Couteur DG, Abernethy DR, Hilmer SN. A pilot randomized clinical trial utilizing the Drug Burden Index to reduce exposure to anticholinergic and sedative medications in older people. Ann Pharmacother. 2010;44(11):1725-1732.

38. Gnjidic D, Le Couteur DG, Kouladjian L, Hilmer SN. Deprescribing trials: methods to reduce polypharmacy and the impact on prescribing and clinical outcomes. Clin Geriatr Med. 2012;28(2):237-253.

39. Patterson SM, Hughes C, Kerse N, Cardwell CR, Bradley MC. Interventions to improve the appropriate use of polypharmacy for older people. Cochrane Database Syst Rev. 2012;5:CD008165.

40. Kaur S, Mitchell G, Vitetta L, Roberts MS. Interventions that can reduce inappropriate prescribing in the elderly: a systematic review. Drugs Aging. 2009;26(12):1013-1028.

41. Durieux P, Trinquart L, Colombet I, et al. Computerized advice on drug dosage to improve prescribing practice. Cochrane Database Syst Rev. 2008;3:CD002894.

42. Garg AX, Adhikari NK, McDonald H, et al. Effects of computerized clinical decision support systems on practitioner performance and patient outcomes: a systematic review. JAMA. 2005;293(10):1223-1238.

43. Hemens BJ, Holbrook A, Tonkin M, et al. Computerized clinical decision support systems for drug prescribing and management: a decision-maker-researcher partnership systematic review. Implement Sci. 2011;6:89.

44. Roshanov PS, Fernandes N, Wilczynski JM, et al. Features of effective computerised clinical decision support systems: meta-regression of 162 randomised trials. BMJ. 2013;346:f657.

45. Kouladjian L, Gnjidic D, Chen T, Hilmer S. PP009 - Development, validation and usability of software to calculate the Drug Burden Index: a pilot study. Clin Ther. 2013;35 Suppl 8:e19.

46. Faure R, Dauphinot V, Krolak-Salmon P, Mouchoux C. A standard international version of the Drug Burden Index for cross-national comparison of the functional burden of medications in older people. $J$ Am Geriatr Soc. 2013;61(7):1227-1228.

47. Hilmer SN, Gnjidic D, Abernethy DR. Drug Burden Index for international assessment of the functional burden of medications in older people. J Am Geriatr Soc. 2014;62(4):791-792.

48. Jones J, Hunter D. Consensus methods for medical and health services research. BMJ. 1995;311(7001):376-380.

49. McLachlan AJ, Hilmer SN, Le Couteur DG. Variability in response to medicines in older people: phenotypic and genotypic factors. Clin Pharmacol Ther. 2009;85(4):431-433.

50. Floroff CK, Slattum PW, Harpe SE, Taylor P, Brophy GM. Potentially inappropriate medication use is associated with clinical outcomes in critically ill elderly patients with neurological injury. Neurocrit Care. May 8, 2014. [Epub ahead of print].

51. American Geriatrics Society 2012 Beers Criteria Update Expert Panel. American Geriatrics Society updated Beers Criteria for potentially inappropriate medication use in older adults. J Am Geriatr Soc. 2012; 60(4):616-631.

52. Gallagher P, Ryan C, Byrne S, Kennedy J, O’Mahony D. STOPP (Screening Tool of Older Person's Prescriptions) and START (Screening Tool to Alert doctors to Right Treatment). Consensus validation. Int J Clin Pharmacol Ther. 2008;46(2):72-83.
53. Gallagher PF, O'Connor MN, O’Mahony D. Prevention of potentially inappropriate prescribing for elderly patients: a randomized controlled trial using STOPP/START criteria. Clin Pharmacol Ther. 2011;89(6): 845-854.

54. Hanlon JT, Schmader KE. The Medication Appropriateness Index at 20: where it started, where it has been, and where it may be going. Drugs Aging. 2013;30(11):893-900.

55. Samsa GP, Hanlon JT, Schmader KE, et al. A summated score for the Medication Appropriateness Index: development and assessment of clinimetric properties including content validity. J Clin Epidemiol. 1994;47(8):891-896.

56. Basger BJ, Chen TF, Moles RJ. Inappropriate medication use and prescribing indicators in elderly Australians: development of a prescribing indicators tool. Drugs Aging. 2008;25(9):777-793.

57. Basger BJ, Chen TF, Moles RJ. Validation of prescribing appropriateness criteria for older Australians using the RAND/UCLA appropriateness method. BMJ Open. 2012;2(5).

58. Carnahan RM, Lund BC, Perry PJ, Pollock BG, Culp KR. The Anticholinergic Drug Scale as a measure of drug-related anticholinergic burden: associations with serum anticholinergic activity. J Clin Pharmacol. 2006;46(12):1481-1486.

59. Ancelin ML, Artero S, Portet F, Dupuy AM, Touchon J, Ritchie K. Nondegenerative mild cognitive impairment in elderly people and use of anticholinergic drugs: longitudinal cohort study. BMJ. 2006;332(7539): 455-459.

60. Han L, Agostini JV, Allore HG. Cumulative anticholinergic exposure is associated with poor memory and executive function in older men. $J$ Am Geriatr Soc. 2008;56(12):2203-2210.

61. Rudolph JL, Salow MJ, Angelini MC, McGlinchey RE. The anticholinergic risk scale and anticholinergic adverse effects in older persons. Arch Intern Med. 2008;168(5):508-513.

62. Ehrt U, Broich K, Larsen JP, Ballard C, Aarsland D. Use of drugs with anticholinergic effect and impact on cognition in Parkinson's disease: a cohort study. J Neurol Neurosurg Psychiatry. 2010;81(2):160-165.

63. Sittironnarit G, Ames D, Bush AI, et al. Effects of anticholinergic drugs on cognitive function in older Australians: results from the AIBL study. Dement Geriatr Cogn Disord. 2011;31(3):173-178.

64. Boustani M, Campbell N, Munger S, Maidment I, Fox C. Impact of anticholinergics on the aging brain: a review and practical application. Aging Health. 2008;4(3):311-320.

65. Linjakumpu T, Hartikainen S, Klaukka T, Koponen H, Kivela SL, Isoaho R. A model to classify the sedative load of drugs. Int J Geriatr Psychiatry. 2003;18(6):542-544.

66. Linjakumpu TA, Hartikainen SA, Klaukka TJ, et al. Sedative drug use in the home-dwelling elderly. Ann Pharmacother. 2004;38(12): 2017-2022.

67. Sloane P, Ivey J, Roth M, Roederer M, Williams CS. Accounting for the sedative and analgesic effects of medication changes during patient participation in clinical research studies: measurement development and application to a sample of institutionalized geriatric patients. Contemp Clin Trials. 2008;29(2):140-148.

68. Boudreau RM, Hanlon JT, Roumani YF, et al. Central nervous system medication use and incident mobility limitation in community elders: the Health, Aging, and Body Composition study. Pharmacoepidemiol Drug Saf. 2009;18(10):916-922.

69. Hanlon JT, Boudreau RM, Roumani YF, et al. Number and dosage of central nervous system medications on recurrent falls in community elders: the Health, Aging and Body Composition study. J Gerontol A Biol Sci Med Sci. 2009;64(4):492-498.

70. Wright RM, Roumani YF, Boudreau R, et al. Effect of central nervous system medication use on decline in cognition in community-dwelling older adults: findings from the Health, Aging And Body Composition Study. J Am Geriatr Soc. 2009;57(2):243-250.

71. National Prescribing Service Limited. Antipsychotic Monitoring Tool. Surry Hills, Australia: 2014. Available from: http://www.nps.org.au/__data/assets/pdf_file/0015/130326/ NPS_Antipsychotic_Monitoring_Tool.pdf. Accessed April 16, 2014. 
72. Kersten H, Molden E, Tolo IK, Skovlund E, Engedal K, Wyller TB. Cognitive effects of reducing anticholinergic drug burden in a frail elderly population: a randomized controlled trial. J Gerontol A Biol Sci Med Sci. 2013;68(3):271-278.

73. Lertxundi U, Domingo-Echaburu S, Hernandez R, Peral J, Medrano J. Expert-based drug lists to measure anticholinergic burden: similar names, different results. Psychogeriatrics. 2013;13(1):17-24.

74. Alldred DP. Deprescribing: a brave new word? Int J Pharm Pract. 2014;22(1):2-3.

75. Frank C. Deprescribing: a new word to guide medication review. CMAJ. 2014;186(6):407-408.

76. Woodward MC. Deprescribing: achieving better health outcomes for older people through reducing medications. J Pharm Pract Res. 2003 ; 33:323-328.

77. He ZK, Ball PA. Can medication management review reduce anticholinergic burden (ACB) in the elderly? Encouraging results from a theoretical model. Int Psychogeriatr. 2013;25(9):1425-1431.

78. West T, Pruchnicki MC, Porter K, Emptage R. Evaluation of anticholinergic burden of medications in older adults. J Am Pharm Assoc. 2013; 53(5):496-504.

79. Gould RL, Coulson MC, Patel N, Highton-Williamson E, Howard RJ. Interventions for reducing benzodiazepine use in older people: metaanalysis of randomised controlled trials. Br J Psychiatry. 2014;204(2): 98-107.

80. Budnitz DS, Lovegrove MC, Shehab N, Richards CL. Emergency hospitalizations for adverse drug events in older Americans. $N$ Engl J Med. 2011;365(21):2002-2012.

81. Tannenbaum C, Martin P, Tamblyn R, Benedetti A, Ahmed S. Reduction of inappropriate benzodiazepine prescriptions among older adults through direct patient education: the EMPOWER cluster randomized trial. JAMA Intern Med. 2014;174(6):890-898.

82. Joester J, Vogler CM, Chang K, Hilmer SN. Hypnosedative use and predictors of successful withdrawal in new patients attending a falls clinic: a retrospective, cohort study. Drugs Aging. 2010;27(11):915-924.

83. Lonnroos E, Gnjidic D, Hilmer SN, et al. Drug Burden Index and hospitalization among community-dwelling older people. Drugs Aging. 2012;29(5):395-404.

84. Lowry E, Woodman RJ, Soiza RL, Hilmer SN, Mangoni AA. Drug Burden Index, physical function, and adverse outcomes in older hospitalized patients. J Clin Pharmacol. 2012;52(10):1584-1591.
85. Swannick G. Prescribing information. eMIMS (CD-ROM). St Leonards Australia: Monthly Index of Medical Specialties.

86. Bosboom PR, Alfonso H, Almeida OP, Beer C. Use of potentially harmful medications and health-related quality of life among people with dementia living in residential aged care facilities. Dement Geriatr Cogn Dis Extra. 2012;2(1):361-371.

87. Hilmer SN, Mager DE, Simonsick EM, et al. Drug Burden Index score and functional decline in older people. Am J Med. 2009;122(12): 1142-1149.

88. Kashyap M, Belleville S, Mulsant BH, et al. Methodological challenges in determining longitudinal associations between anticholinergic drug use and incident cognitive decline. J Am Geriatr Soc. 2014;62(2): 336-341.

89. Narayan SW, Hilmer SN, Horsburgh S, Nishtala PS. Anticholinergic component of the Drug Burden Index and the anticholinergic drug scale as measures of anticholinergic exposure in older people in New Zealand: a population-level study. Drugs Aging. 2013;30(11):927-934.

90. Nishtala PS, Narayan S, Wang T, Hilmer SN. Associations of Drug Burden Index with falls, general practitioner visits, and mortality in older people. Pharmacoepidemiol Drug Saf. 2014;23(7):753-758.

91. Cumming RG, Handelsman D, Seibel MJ, et al. Cohort profile: the Concord Health and Ageing in Men Project (CHAMP). Int J Epidemiol. 2009;38(2):374-378.

92. Best O, Gnjidic D, Hilmer SN, Naganathan V, McLachlan AJ. Investigating polypharmacy and Drug Burden Index in hospitalised older people. Intern Med J. 2013;43(8):912-918.

93. Dispennette R, Elliott D, Nguyen L, Richmond R. Drug Burden Index score and anticholinergic risk scale as predictors of readmission to the hospital. Consult Pharm. 2014;29(3):158-168.

94. Han L, McCusker J, Cole M, Abrahamowicz M, Primeau F, Elie M. Use of medications with anticholinergic effect predicts clinical severity of delirium symptoms in older medical inpatients. Arch Intern Med. 2001;161(8):1099-1105.

95. Chew ML, Mulsant BH, Pollock BG, et al. Anticholinergic activity of 107 medications commonly used by older adults. J Am Geriatr Soc. 2008;56(7):1333-1341.
Clinical Interventions in Aging

\section{Publish your work in this journal}

Clinical Interventions in Aging is an international, peer-reviewed journal focusing on evidence-based reports on the value or lack thereof of treatments intended to prevent or delay the onset of maladaptive correlates of aging in human beings. This journal is indexed on PubMed Central, MedLine,

\section{Dovepress}

CAS, Scopus and the Elsevier Bibliographic databases. The manuscript management system is completely online and includes a very quick and fair peer-review system, which is all easy to use. Visit http://www.dovepress. com/testimonials.php to read real quotes from published authors. 